Geophysical and Astrophysical Fluid Dynamics

Vol. 00, No. 00, 00 Month 2012, 1-14

\title{
Some statistical properties of three-dimensional zonostrophic turbulence
}

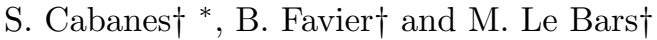 \\ †CNRS, Aix Marseille Univ, Centrale Marseille, IRPHE, Marseille, France. \\ (v4.4 released October 2012)
}

\begin{abstract}
We conduct in-depth analysis of statistical flow properties from direct numerical simulations that reproduce gas giants macroturbulence, namely large-scale zonal winds. Our numerical model has been specifically designed to simulate a recent laboratory device that reports zonal jets in the configuration of deep turbulent planetary layers (Cabanes et al. 2017). In this framework, the so-called zonostrophic regime is achieved when large topographical variations of the fluid layer combine with rapid rotation in a well developed three-dimensional (3D) turbulent flow. At steady state, strongly energetic, zonally dominated, large-scale axisymmetric structures emerge scaling with Rhines' theoretical scale. This model differs from the shallowlayer scenario where the flow is confined to a quasi-two-dimensional (2D) fluid shell and the anisotropic $\beta$ effect arises from latitudinal variation of the Coriolis force. Thus, we aim to reveal, in the specific framework of the deep-layer scenario, signatures of the zonostrophic regime and of a $\beta$-topography in statistical flow properties. To do so, we run two large-scale 3D direct numerical simulations in a cylindrical geometry of a highly turbulent and rapidly rotating flow. These two simulations use similar set of parameters but with and without topographical $\beta$-effect. We propose a comparative phenomenological description of the temporal and spatial statistics of the three components of the velocity field. Interestingly, we report that peculiar correlations occur between the vertical and radial flow components when a $\beta$-topography is imposed and show a feature possibly due to a zonostrophic dynamics in 3D frequency spectra. These results suggest the development of new tools to remotely investigate gas giants zonal winds by extracting statistical flow properties from direct observations. Ultimately our analysis may support the relevance of the deep models in the study of prevalent features of planetary dynamics.
\end{abstract}

Keywords: Zonostrophic turbulence, zonal jets.

\section{Introduction}

The strong east-west winds on the gas giants, such as Jupiter (Bagenal et al. 2006) and Saturn (Del Genio et al. 2009), are unmistakable markers of powerful underlying dynamics, inaccessible to direct measurements. Understanding how these intense zonal winds emerge and interact with deeper planetary envelops, is a major scientific challenge for ongoing spacecraft missions as well as for numerical and laboratory studies. While classical homogeneous three-dimensional (3D) turbulence leads to a direct cascade of the energy, turbulence theory predicts that large scale structures naturally emerge from small-scale motions in two-dimensional (2D) systems. This well-known phenomenon is called an inverse energy cascade (Kraichnan 1967). Consequently, macro-turbulence on Jupiter has been interpreted to result from the inverse energy cascade within the quasi-2D shallow troposphere (Cho and Polvani 1996) (i.e., the planetary weather layer). Similar assumptions are used in global circulation models designed to simulate large-scale circulations observed in the Earth's atmosphere and oceans. It is then common to invoke that the vertical dimension (along the planetary radius) is much smaller than any typical horizontal scale, thus reducing simulations to a quasi-2D configuration (e.g. solving shallow-water equations on a planetary spherical surface). However, recent studies have revived the possibility to generate Jupiter like jets from a 3D convective envelope, extending through the molecular layer between the surface 
weather layer and the deeper dynamo region (Christensen 2001, Heimpel et al. 2005, Kaspi et al. 2009, Heimpel et al. 2016, Cabanes et al. 2017). These studies invoke that rapid planetary rotation leads to flow invariance along the planetary spin axis. This dynamical confinement due to a dominant Coriolis acceleration forces the flow to be invariant along the rotation axis and sets the required conditions for the inverse cascade to feed large scale structures. This duality between 2D and 3D dynamics, inverse and direct cascades, is at the core of fundamental geophysical fluid dynamics (Pouquet and Marino 2013). In both shallow and deep configurations, the dynamics is quasi-2D, namely depth invariant, and large scales develop on the horizontal flow components. In the shallow model, horizontal is defined by the spherical planetary surface and depth is along the planetary radius. In the deep model, the relevant horizontal and depth components are defined to be respectively normal and along the planetary spin axis. This defines a complete framework to study the specificity of the shallow and deep-layer scenarios.

In the specific context of planetary systems, the large scale structures formed by quasi-2D flows display an additional zonal anisotropy in the form of east-west dominant motions. Such features appear in spherical geometry where the boundary curvature of planetary fluid layers preferentially channels energy into the large-scale zonal motions via the so-called $\beta$-effect (Vallis and Maltrud 1993). Large-scale drag eventually halts the inverse energy cascade that propagates up to the Rhines' length scale $L_{R h}=\pi \sqrt{2 U / \beta}$, and sets the characteristic width of zonal jets (Rhines 1975, Sukoriansky et al. 2007). Here $U$ is the root-mean-square (rms) fluid velocity. In shallow turbulence, where quasi-bidimensionality results from spatial confinement, zonal anisotropisation arises from latitudinal variation of the Coriolis force with $\beta=2 \Omega \cos \theta / R$, where $\Omega$ is the rotation rate, $\theta$ is the latitude and $R$ is the radius of the planet. In deep turbulence, where quasi-bidimensionality results from strong anisotropy induced by the Coriolis force, zonal anisotropy emerges from variations of the axial fluid column height $h$ with cylindrical radius $r$, with $\beta=(2 \Omega / h) d h / d r$. Both scenarios gather all suitable physical ingredients and zonal jets develop, provided that the Coriolis force dominates over fluid inertia (i.e, low Rossby number Ro), which in turn, must dominate over viscous dissipative effects (i.e., large Reynolds number $R e$ and small Ekman number $E$ ). Depending on the details of the system considered, the large-scale jets can remain weak and meandering like in the Earth's oceans (Maximenko et al. 2005), or ultimately reach the so-called zonostrophic regime where zonal energy dominates over turbulent fluctuations. These differing regimes of jet flow behaviors can be characterized in terms of the zonostrophy index $R_{\beta}$ (Galperin et al. 2010). This index is the ratio of the Rhines scale and the transitional scale $L_{\beta} \approx\left(\epsilon / \beta^{3}\right)^{1 / 5}$ above which the $\beta$-effect affects the energy cascade, with $\epsilon$ being the inverse energy transfer rate through the system. Simplistically, $R_{\beta} \approx L_{R h} / L_{\beta}$ may be regarded as a measure of how strongly anisotropic the turbulent flow may be. Zonostrophic flows are argued to exist at $R_{\beta} \geq 2.5$ (Galperin et al. 2006, Sukoriansky et al. 2007). Jovian mid-latitude jets exceed this threshold (Galperin et al. 2014) with a typical value $R_{\beta} \approx 5$, while Earth's oceans are limited to $R_{\beta} \approx 1.5$ (Galperin et al. 2006). Strongly zonostrophic regime is the focus of the present paper.

As of now, although these shallow and deep models have been systematically challenged, there has been no successful attempt to contrast their relevance in the study of the prevalent features of planetary dynamics. Today, new observations from the ongoing Juno NASA's mission pave the way to conduct in-depth analyzes of Jupiter's flow properties. The upcoming challenge will be to identify, using the measurements of the Jovian anisotropic turbulent flow, typical signatures of the shallow and deep turbulent scenarios. For that purpose, laboratory and numerical models for planetary jets are now requested to come up with a full description 
of turbulent flow properties in the framework of zonostrophic systems. However, planetary-like jets have been reproduced mainly in numerical models with simplifying assumptions and limitations (Cho and Polvani 1996, Jones and Kuzanyan 2009). Jets dynamics is sometimes studied in idealized numerical models solving, in the framework of $\beta$-plane approximation, for 2D quasi-geostrophic turbulence in single (Scott and Dritschel 2012, 2013) or multiple (Berloff et al. 2011) planetary layers in vertical. Attractive alternative studies equally retrieve basic jets properties, in idealized $2 \mathrm{D}$ system with $\beta$-plane, by modeling kinetic energy transfer from small turbulent scales to large zonal scales using stochastic methods or closure models (Bouchet et al. 2013, Marston et al. 2016). It is then common, when a planetary like spherical configuration is considered, that the shallow scenario is modeled solving for three-dimensional general circulation model (GCM) of a thin shell in the outer atmosphere, assuming weak depth variations along the planetary radius (Schneider and Liu 2009). Numerical studies designed to simulate the deep model (in which rotation leads to axial flow invariance of a 3D-turbulent system) are only considered using hyper-diffusion or stress-free boundary conditions (Christensen 2001, Heimpel et al. 2005, 2016). Note that, contrary to shallow-layer models, where depth invariance is assumed and not produced by the dynamics, vertical motions including wave motions may play an important role in deep-layer 3D turbulent models (see e.g. Yarom and Sharon 2014). Previous laboratory studies (see for instance the recent studies by Di Nitto et al. 2013, Smith et al. 2014, Zhang and Afanasyev 2014, Read et al. 2015, Galperin et al. 2016) have reached weakly zonostrophic regimes (with $R_{\beta}<2.5$ ).

In the present paper, we extend the analysis initiated by Cabanes et al. (2017) of the first laboratory/numerical model generating planetary-like zonal jets in a deep 3D-layer. In addition to a large-scale rotating experiment, Cabanes et al. (2017) performed a 3D turbulent direct numerical simulation (DNS) with a cylindrical $\beta$-topography designed to emulate part of a 3D convective planetary layer. Contrary to previous numerical simulations aforementioned, 3D Navier-Stokes equations have been solved in realistic experimental conditions using no-slip boundary conditions. Deep-seated jets have been observed with similar flow properties in the numerical simulation and in its laboratory realization. The strength and scale of this multiple jets system have been shown to be comparable relative to those observed on the gas planets. In the direct continuity of these first results, we propose here a phenomenological description of flow statistics in this high resolution direct numerical simulation referred to as DNS1. In order to investigate the specific role of the $\beta$-topography and its impact on turbulent flow properties, we run a second DNS using similar dynamical conditions but without layer depth variation (labeled as DNS2). Spatial and temporal spectral flow description, comparatively in the $\beta$ and non- $\beta$ simulations, allows us to extract the signatures of the zonostrophic turbulence in a fully 3D system with realistic dissipative effects. In particular, we evidence the peculiar correlation of the vertical and radial flow components under topographic $\beta$-effect and report, for the first time, a signature of the zonostrophic regime in frequency spectra. Our findings may open new horizons to remotely investigate the dynamics of gas giants' zonal winds using phenomenological description of turbulent flow properties.

\section{Numerical setups}

We consider the motion of an incompressible fluid with constant kinematic viscosity $\nu$ and constant density $\rho$. The fluid is contained inside a circular cylinder of radius $R$ rotating with a constant rate $\Omega$ around the vertical axis. In our first model DNS1 (see figure 1(a)), the vertical extend of the fluid layer $h(r)$ is axisymmetric and corresponds to the analytical paraboloid 
shape of a free surface that depends on the rotation rate and on the gravitational acceleration $g$ given by

$$
h(r)=h_{0}-\frac{\Omega^{2} R^{2}}{4 g}\left(1-2 \frac{r^{2}}{R^{2}}\right),
$$

where $h_{0}$ is the fluid height in the absence of rotation. This upper paraboloidal surface is assumed to be stress-free whereas both side and bottom boundaries are no-slip. This appropriately models the experimental setup described in Cabanes et al. (2017) and also mimics topographic variations near planetary poles. The center, with minimum fluid height, corresponds to the pole, while the edge of the domain corresponds to lower latitudes. The simulation is set so that the nature of the $\beta$ parameter (i.e., cylindrically-radial topographic variations) and the large dynamical jets' aspect ratio (i.e., vertical jets' depth larger than their horizontal width $L_{R h}$ ) reproduce a deep planetary layer. We have also run a second DNS (labeled as DNS2 in the following), dynamically similar to the first run DNS1 but this time with a top flat stress-free surface. All parameters characterizing the system remain the same, but the uniform layer depth is now $h_{0}$, which corresponds to the limit case $\beta=0$. This second case is run as a reference, as there is no topographic effect in that case. It is also close to other rotating turbulence experiments, as for instance by Yarom and Sharon (2014). For the two cases, energy is injected through a small-scale steady horizontal volumic force corresponding to Taylor-Green vortices. The forcing is given by the following formula:

$$
\boldsymbol{F}= \begin{cases}{\left[\begin{array}{c}
A S(r) \sin \left(\pi k_{f} x\right) \cos \left(\pi k_{f} y\right) \sin \left(\pi k_{f} z\right) \\
A S(r) \cos \left(\pi k_{f} x\right) \sin \left(\pi k_{f} y\right) \sin \left(\pi k_{f} z\right) \\
0
\end{array}\right]} & \text { if } \quad 0 \leq z \leq 1 / k_{f}, \\
0 & \text { elsewhere. }\end{cases}
$$

The function $S(r)$ is given by

$$
S(r)=\frac{1}{2}[\tanh (60(r-0.15))-\tanh (60(r-0.92)],
$$

and is used to avoid the generation of intense vortical structures close to the outer boundary and at the center of the cylindrical domain. $A$ is the ratio of the force amplitude to the centrifugal acceleration and can be seen as a forcing Rossby number $\left(A=2 \times 10^{-3}\right.$ in the following). We focused on the configuration where the forcing is at relatively small scales (i.e., with a typical horizontal wavenumber $k_{f}=12$ ) to ensure scale separation between the injection of the energy and the jet scale, but not too small so that the local Reynolds number is still large. Note that it is very demanding numerically to allow for a scale separation between the injection and the large-scale flows, while solving at the same time for the inertial range associated with the three dimensional direct cascade. Contrary to previous numerical simulations, designed to model deep-seated jets with negligible role of viscous dissipation, we solve for a viscous flow with realistic no-slip boundary conditions applied on the bottom and side walls. In particular, multiple banded flows are not found in the most recent, high-resolution models that couple the molecular envelope to the deeper dynamo region. In these models, bottom dissipation damps the higher-latitude deep jets out of existence (Heimpel and Gómez Pérez 2011, Gastine et al. 2014, Jones 2014). Here, long-lived jets can persist even under conditions including viscous dissipation and no-slip conditions.

We solve the Navier-Stokes equations in the rotating frame using the spectral element solver Nek5000 (Fischer et al. 2007, 2008). Variables are decomposed into Lagrange polynomials based on Gauss-Lobatto-Legendre quadrature points inside hexahedral elements. For both simulations, we use polynomials of order 11 so that each element contains $12^{3}$ grid points. The original mesh corresponds to a full circular cylinder of unit radius and unit height with 53760 elements. In order to match the upper paraboloidal boundary in the DNS1 model, we 
a
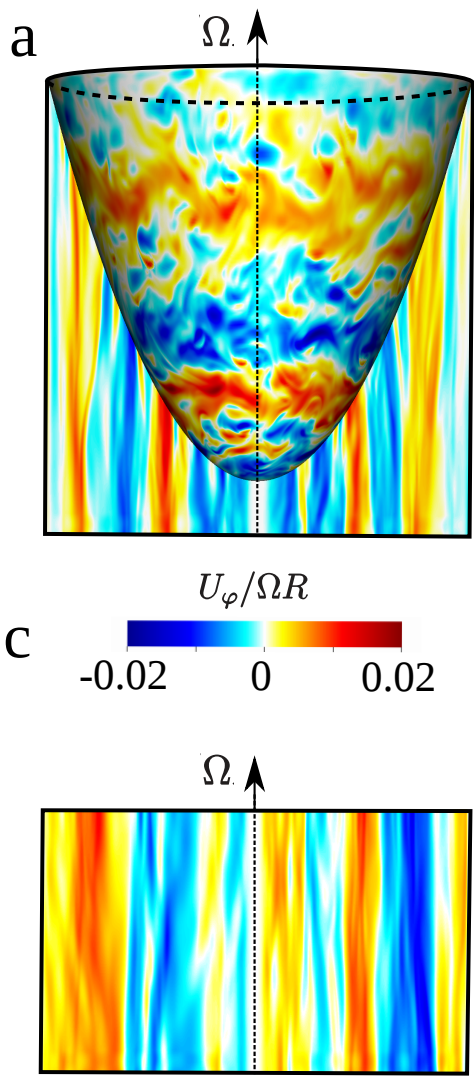

$\mathrm{b}$
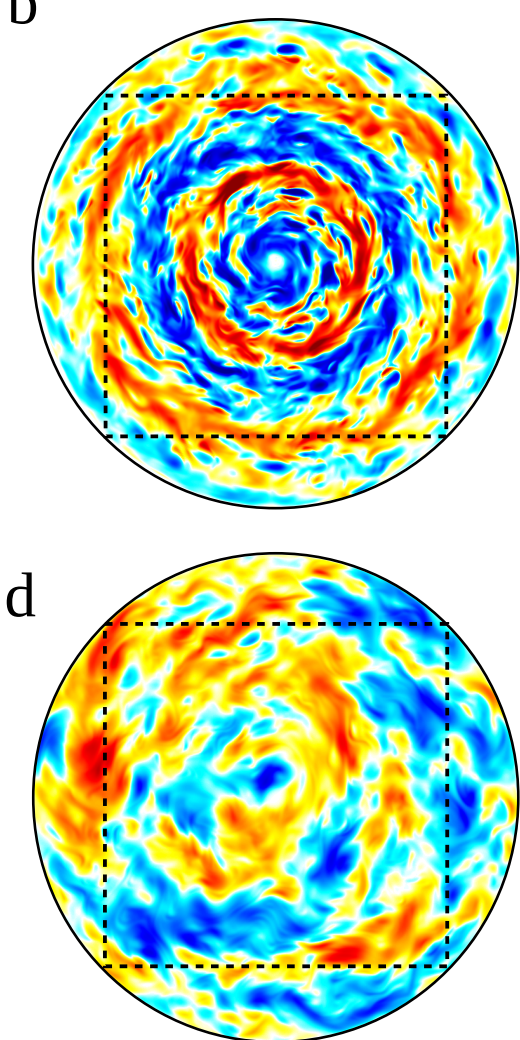

Figure 1. Sidecut and top view of DNS1 (a-b) and DNS2 (c-d) after $t=1000$ rotations showing the instantaneous non-dimensional azimuthal velocity field at $E=5 \times 10^{-7}, R o=2 \times 10^{-3}$ and $R e=4 \times 10^{3}$, based on the rms flow velocity and the averaged fluid depth. The dashed squares in (b) and (d) show the location where spectral analysis is performed (see section 4 ).

\section{Global properties and visualizations}

The time evolutions of the rms values of all three velocity cylindrical components $\left(u_{r}, u_{\phi}, u_{z}\right)$ are shown in figure 2. After a very short transient phase where all components grow rapidly (few rotation times), a secondary transient growth occurs in both cases on much longer timescales (typically several hundreds of rotation times) until complete saturation eventually occurs. In case DNS1, this slow secondary growth only affects the azimuthal component whereas radial and vertical components are similar and saturate quickly around a lower 

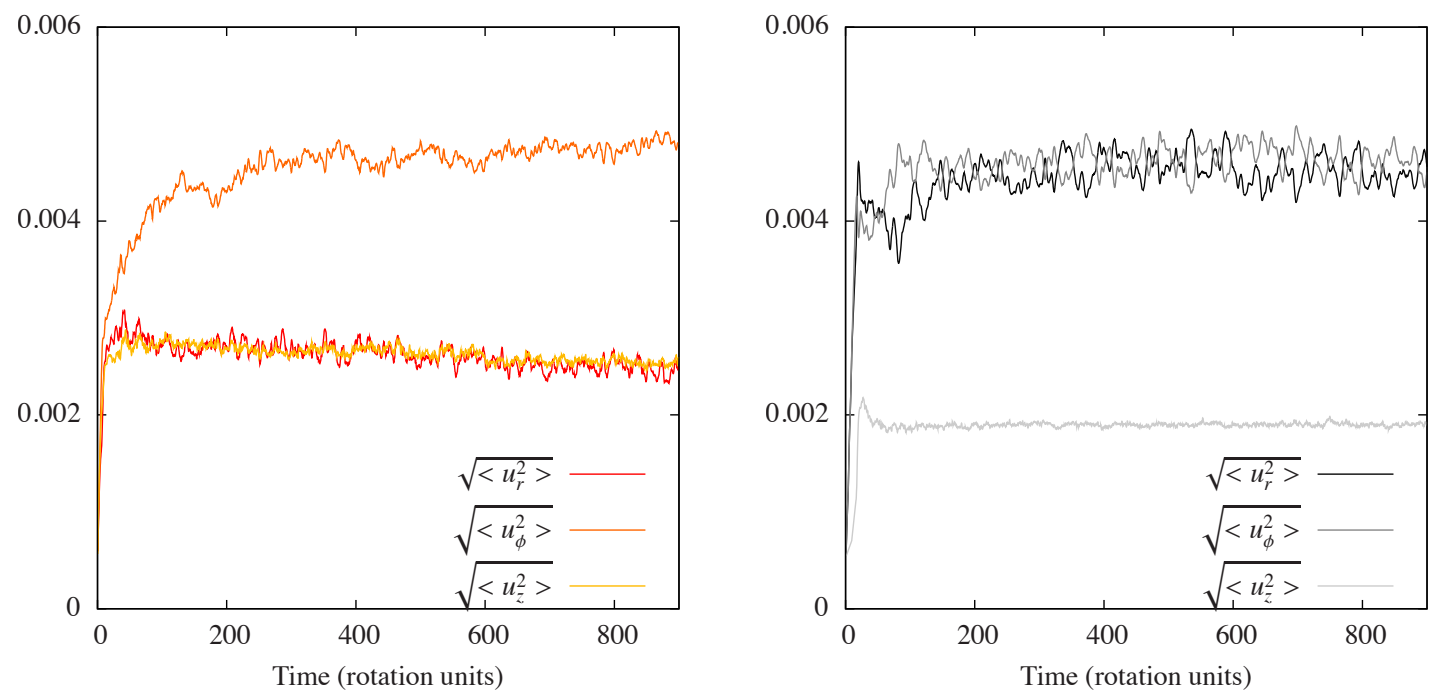

Figure 2. Time evolution of the rms velocity components for the case DNS1 with topographical effects (left) and the case DNS2 without topographical effects (right). (Colour online)

value. In case DNS2, both horizontal components are similar and saturate at large amplitude whereas the vertical component behaves differently and saturates at lower amplitude. This is the first clear distinction between the so-called zonostrophic regime when compared with the regular rapidly-rotating regime: the anisotropy induced by the rotation does not affect all velocity components in the same way. Without topographical effects, the horizontal flow remains isotropic whereas a clear zonal anisotropy is observed when topographical effects are important.

We show in figure 1 sidecut and top views, after 900 rotations, of the instantaneous zonal velocity field. For case DNS1, the flow displays five prograde/retrograde jets (red/blue), with strong, instantaneous signature, and with similar properties to those observed on the gas planets (see Cabanes et al. 2017, for details). More than 50\% of the total surface kinetic energy concentrates in the zonal velocity component. This large scale surface flow is seated on a deep, nearly invariant geostrophic flow, confirming that the system is quasi-2D under dynamical conditions $(R o \ll 1)$. Case DNS2 is also nearly invariant along the rotation axis but does not display such axisymmetry. Large-scale flows are still apparent but irregularly distributed across the horizontal plane.

More quantitatively, the establishment of the zonostrophic regime in DNS1 has been argued in detail by Cabanes et al. (2017) and can be summed-up as follows: using the rms of the total velocity $U_{r m s}^{T}$, the estimate of the Reynolds $R e=U_{r m s}^{T} h_{o} / \nu=10^{3}$ and the Rossby $R o=U_{r m s}^{T} / 2 \Omega h_{o}=10^{-3}$ non-dimensional numbers attests that parameters range achieved in DNS1 and DNS2 is suitable to grow large-scale structures in 3D-turbulent flows. The additional $\beta$-topography in DNS1 leads to the existence of a meaningful zonostrophic inertial range whose width is estimated by the zonostrophic index $R_{\beta} \approx L_{R h} / L_{\beta}$. The total $\mathrm{rms}$ velocity $U_{r m s}^{T}$ and the mean value $\beta=73 \mathrm{~m}^{-1} \mathrm{~s}^{-1}$ are used to estimate Rhines wavenumber 
$k_{R h}=1 / L_{R h}$. The transitionnal wavenumber $k_{\beta} \approx 1 / L_{\beta}$ is defined using a revised expression $k_{\beta}=0.73\left(\beta^{3} / \epsilon\right)^{1 / 5}$ found in Galperin et al. (2014) and confirmed in Cabanes et al. (2017). The upscale energy transfer rate is estimated as $\epsilon \simeq\left(U_{r m s}^{T}\right)^{2} / 2 \tau_{E}$ based on the total kinetic energy, the Ekman spinup-time $\tau_{E}=h_{o} / \sqrt{\nu \Omega}$ (Read et al. 2015) and it has been shown in Cabanes et al. (2017) that this estimate for $\epsilon$ gives good predictions of the inverse energy transfer rate recovered in kinetic energy spectra. The resulting zonostrophic inertial range, delimited by typical wavenumbers $k_{R h}$ and $k_{\beta}$, is reported figure 3(a). A typical estimate for the zonostrophic index gives $R_{\beta} \approx 3.4$ (this index goes up to 3.7 in the laboratory, more calculation details are given in Cabanes et al. (2017)). This value significantly exceeds the 2.5 threshold required to set the zonostrophic regime.

Let us first discuss the zonal spectrum computed from spectral decomposition of the axisymmetric component of the flow, here based on the time and azimuthal average of the zonal velocity. As shown in figure $3 \mathrm{a}$, we recover the theoretical prediction $\overline{E_{\varphi}}\left(k_{\perp}\right)=C_{\varphi} \beta^{2} k_{\perp}^{-5}$, with $k_{\perp}$ the orthogonal wavenumber, perpendicular to the azimuthal component, and $C_{\varphi}=2$ the zonal constant (Sukoriansky et al. 2002, 2007, Galperin et al. 2014). To distinguish between the axisymmetric and non-axisymmetric components of the flow, one defines the origine to be the rotation spin axis for spectral analysis. The axisymmetric flow is then a one dimensional profile along the radius in our cylindrical geometry. Following this approach, the orthogonal wavenumber corresponds to the radial wavenumber, $k_{\perp}=k_{r}$. We note here that cylindrical harmonic decomposition of the flow would have been more appropriated than time and azimuthal average of the velocity field to compute the axisymmetric flow component. However, to facilitate comparison with spectral analysis in the laboratory reported in Cabanes et al. (2017) (which was performed in Cartesian coordinates due to the lack of a complete cylindrical field) we decided to adopt the same data processing for statistical description of the flow. We ascertain however that both approaches lead to comparable results. Thus, consistently with aforementioned studies (Sukoriansky et al. 2002, 2007, Galperin et al. 2014), kinetic energy distribution follows a $k_{r}^{-5}$ slope on both sides of the transitional wavenumber, it achieves maximum values at large scales and drops at wavenumbers smaller than the theoretical Rhines wavenumber. This characteristic spectrum of zonostrophic flows has been commented in details by Cabanes et al. (2017) and confirms that the present model is comparable with Jupiter's jets flow. In the flat geostrophic case (DNS2), we verify that energy of the time and azimuthal average of the zonal velocity is two orders of magnitude smaller than in case DNS1 and that Rhines scale is not a relevant scale in the dynamics. DNS2 is in the rapidly-rotating but non-zonostrophic regime $(R o \ll 1, R e \gg 1$ and $\beta=0)$. These two simulations set the way to investigate the role of the topographic $\beta$-effect in 3D-turbulent rotating flows and to characterize the specificity of the deep-layer scenario. Based on Cabanes et al. (2017), who attested the establishment of a zonostrophic regime in DNS1, we now extend the study to a full spatial and temporal statistical characterization of the three components of velocity fluctuations.

\section{Azimuthal fluctuations}

Because the velocity field is quasi-invariant with depth, we restrict our flow description to a horizontal plane normal to the rotation axis, specifically $z=0.15$. Figure 1 (b) and (d) shows horizontal cuts of the zonal velocity. At steady state, we observe large scale non-axisymmetric structures when the $\beta$-topography is absent (see DNS2 figure $1(\mathrm{~d})$ ). On the contrary, a paraboloidal surface preferentially channels energy into the zonal direction leading to prograde/retrograde jets formation (see DNS1 figure 1(b)). To facilitate the spectral 
$\mathrm{a}$

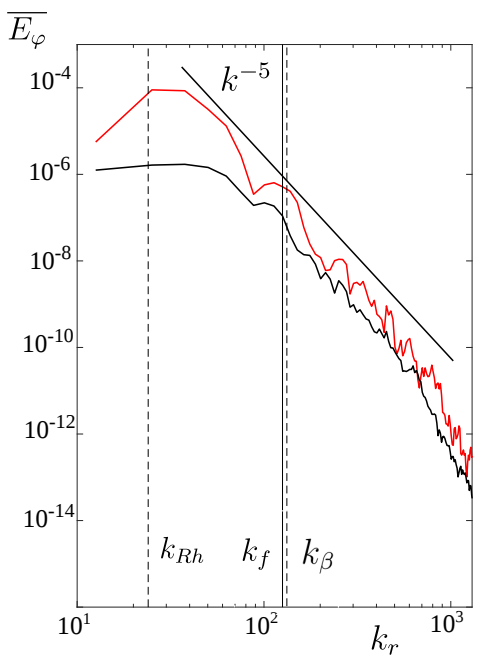

b

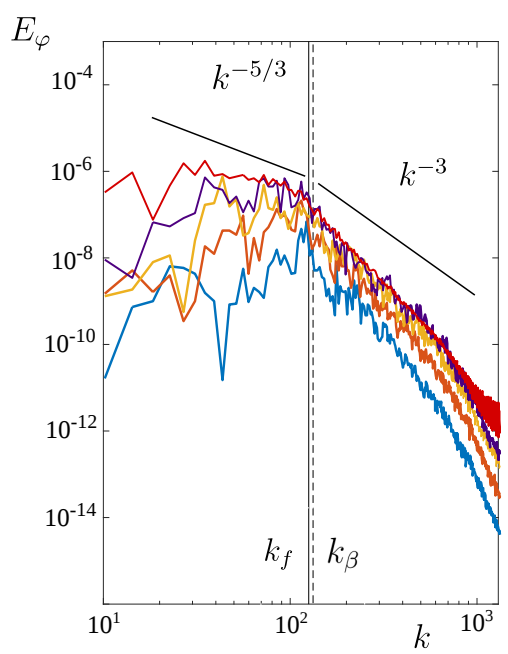

C

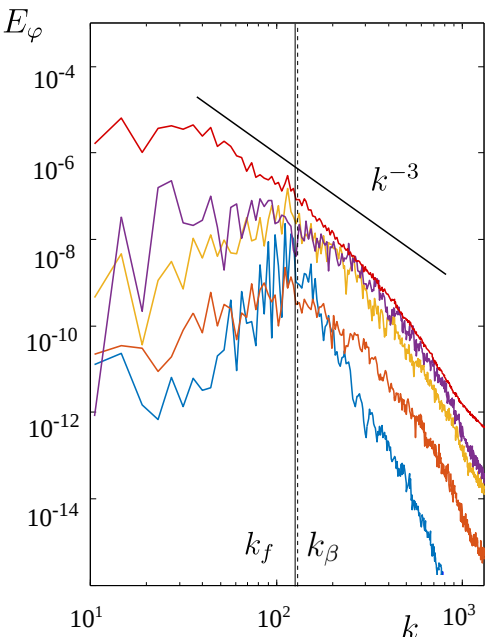

Figure 3. (a) Kinetic energy spectra of the time and azimuthal averaged zonal velocity field for DNS1 (red solid line) and DNS2 (black solid line). The theoretical energy spectrum $\overline{E_{\varphi}}\left(k_{r}\right)$ for the zonostrophic regime (Sukoriansky et al. 2007) is represented by a black straight line as a a function of the radial wavenumber $k_{r}$. Theoretical values of the Rhines $k_{R h}$, the transitional $k_{\beta}$ and the injection $k_{f}$ wavenumbers are respectively shown as dashed grey, vertical dashed black, and solid lines. Typical value of $k_{\beta}$ is estimated using $\epsilon \simeq\left(U_{r m s}^{T}\right)^{2} / 2 \tau_{E}$, with $\tau_{E}$ the Ekman spinup-time. (b-c) $2 \mathrm{D}$ zonal kinetic energy spectra $E_{\varphi}$ as a function of the total wavenumber $k$. Power spectra are given at different times $t=0,5,8,31$ and 1000 rotations (blue, orange, yellow, purple and red) for DNS1 (b) and DNS2 (c). We also report the injection wavenumber $k_{f}$ as a vertical line and the transitional wavenumber $k_{b e t a}$ as a vertical dashed line. (Colour online)

Figure 3(b) and (c) show instantaneous 2D-spectra of zonal fluctuations, at different time steps from initial forcing to steady state. Fluctuations are obtained by subtracting the time averaged flow to the instantaneous velocity field in our Cartesian grid. We ascertain here that temporal and azimuthal average are equivalent in our Cartesian grid to extract the axisymmetric flow component. At initial time, energy is injected from basal forcing at scale $k_{f}$ (blue curves). Temporal evolution of the zonal spectra clearly shows how energy transits in time from initial injection at small-scale to the formation of large-scale structures. With topographic $\beta$-effect, narrow but clear inertial ranges develop on both sides of the forcing scale following the double cascade scenario predicted by Kolmogorov-Batchelor-Kraichnan (KBK) (Kraichnan 1967) in 2D-turbulence (figure 3(b)). The existence of two inviscid invariants (energy and enstrophy) of the 2D Navier-Stokes equations implies an inverse cascade of kinetic energy to large scales $\left(k<k_{f}\right)$ with a power-law of $E(k) \propto k^{-5 / 3}$, and a direct cascade of enstrophy to small scales $\left(k>k_{f}\right)$ inducing the power-law $E(k) \propto k^{-3}$. These features are well retrieved in the instantaneous azimuthal velocity of our 3D system, which is dynamically quasi-2D because of the effect of strong rotation. This double cascade scenario has previously been observed in 2D turbulence numerical simulations (Boffetta and Musacchio 2010) as well as in forced rotating flows (Campagne et al. 2014). It is now reported in the present work when topographical effects are dominant, although we are limited by the relatively small scale separation between the energy injection scale and the container's typical length scale. Indeed, we note that previous statistical analyses of the zonostrophic regime have been reported in 
studies solving for 2D vorticity equations (Galperin et al. 2010, Sukoriansky et al. 2012). In these studies, the injection wavenumber $k_{f}$ is much larger than the transitional wavenumber $k_{\beta}$ (namely $k_{f} \gg k_{\beta}$ ). The inverse cascade consequently develops on a much wider inertial range $k<k_{f}$, leading to a $k^{-5 / 3}$ spectrum spreading on both sides of the $k_{\beta}$ wavenumber. In the present work, injection and transitional wavenumbers are approximatively identical, due to numerical constraints, leading the inverse cascade to develop on wavenumbers $k<k_{\beta} \approx k_{f}$ and the direct cascade to develop on wavenumbers $k>k_{\beta} \approx k_{f}$. This lack of scale speration is waiting for extended numerical capacity to increase $k_{f}$, while maintaining turbulence at the injection scale, and finally disclose the dynamics around the transitional wavenumber $k_{\beta}$ in 3D-turbulent zonostrophic flows. Similarly, the $k^{-5}$ slope on wavenumbers $k>k_{\beta}$ observed Figure $3(\mathrm{a})$ is disputable as it occurs beyond the injection wavenumber $k_{f}$.

When no topographic effect is present (figure 3(c)), the inverse cascade still transfers energy to large scale vortices. There is however no equivalent to the Rhines scale so that the cascade continuously feeds large-scale flows that finally saturate due to frictional effect on the lateral and bottom rigid no-slip boundaries. The resulting kinetic energy spectrum is apparently modified compared to the case with topography, and we observe instead a slope closer to $k^{-3}$, altough we cannot be fully conclusive since there is less than a decade available. One possible explanation is the presence of so-called condensates formed by a $2 \mathrm{D}$ turbulence inverse cascade in close or periodic containers (Chertkov et al. 2007) that follow a steeper power-law $E(k) \propto k^{-3}$. Note that in the context of forced rotating turbulence at Rossby and Reynolds number similar to the ones considered here, both $k^{-5 / 3}$ and $k^{-3}$ slopes have been observed in the inverse cascade range depending on the anisotropy of the forcing (Sen et al. 2012, Pouquet et al. 2013). In addition, the large-scale friction due to the side boundary might affect differently the non-axisymmetric flow without topography compared to the dominantly axisymmetric flow in the presence of topography, thus leading to different spectral slopes at large-scales. The reason why this finite size effect does not occur on the azimuthal component of the zonostrophic regime has to be investigated, but may relate to the dynamical, non-rigid, confinement imposed by the Rhines scale (Vallis and Maltrud 1993). Accurate determination of the slope remains to be validated using larger numerical simulations (i.e. larger scale separation) and laboratory experiments.

\section{Radial and vertical fluctuations}

Figure 4(a) reports 2D spectra at steady state of the vertical and radial fluctuations (note that time averaged radial and vertical flows are both negligible) in the horizontal planes displayed in figure 1. With topographic $\beta$, the amplitude of those two components is much smaller than the azimuthal one (consistent with figure 2(a)). Also, both radial and vertical velocity fluctuations are limited to scales that do not exceed Rhines wavenumber. On the contrary, the large scale vortices formed when the top surface is flat have the same signature on the radial and zonal component: we retrieve a $E(k) \propto k^{-3}$ power-law behavior (see figure 3(c) and 4(a)).

Interestingly, with topographic $\beta$, vertical velocity component is slightly more energetic than in the flat case at all scales (dashed lines in figure 4). It traduces that radial motions, when impacting the paraboloidal topography, convert into vertical motions due to the non-penetrative condition of the topographic surface. Consequently, radial and vertical velocities are correlated. To attest this spatial correlation we report in figure 4(b) and (c) the joint probability distribution functions (PDF) of the vertical and radial velocities, with and without topography. Without topography, the PDF plotted in figure 4(c) shows 
that vertical and radial motions are not correlated. Under strong rotation and without topographical variations in a $3 \mathrm{D}$ rapidly-rotating system, the vertical velocity component actually behaves as a quasi-passive scalar. However, when a paraboloidal topography is imposed, the PDF shown in figure 4(b) shows that positive/positive and negative/negative values of the radial/vertical velocities are correlated. In other words, inward (outward) radial motions, when they impact (move away from) the topography, convert into downward (upward) vertical motions. We note that these correlations are maximum for radius where $\beta$ is maximum (not shown). Joint PDF of the other velocity components are not reported in this manuscript as they do not display specific correlations.

a

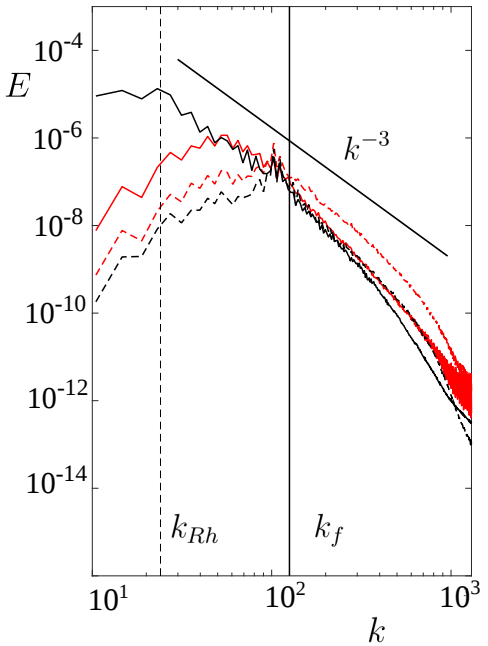

b
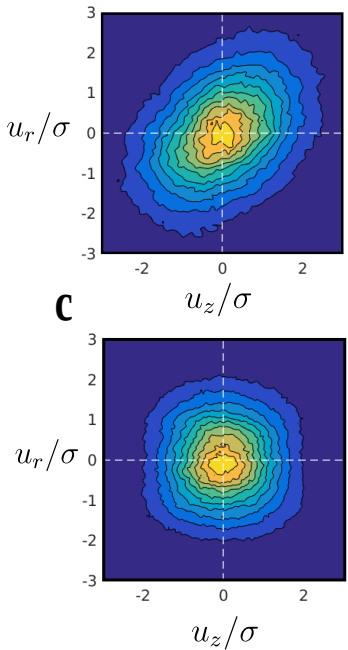

Figure 4. (a) 2D kinetic energy spectra of the radial (solid lines) and vertical (dashed lines) velocity field components, computed from the square grid defined figure 1. Power spectra have been time averaged once steady state is reached. DNS1 and DNS2 are plotted respectively in red and black. $k_{f}$ and $k_{R h}$ are respectively the injection and Rhines wavenumbers. Right: joint probability distribution functions normalized by standard deviation $\sigma$ of the vertical and radial velocity components of the fluctuations for DNS1 (b) and DNS2 (c). (Colour online)

These correlations of the radial and vertical flows are a specificity of 3D-rotating turbulence under topographical $\beta$-effect, as it is the case in 3D-convective planetary layers. Similar flow measurements on gas planets' surface may highlight the dynamics underlying the jets. Note that velocity correlations are obtained from a numerical simulation where the free surface is not deformable. In the laboratory, the iso-pressure paraboloidal free-surface is non-rigid and deformations can occur (typically of a few $\mathrm{mm}$ for maximum radial velocities). Even if these deformations appear to be weak, it may attenuate the observed correlation. Direct measurements of velocity correlations in the laboratory will be further required to confirm our results.

\section{Frequency spectra}

We now focus on the frequency spectra and on the possible signatures associated with the observed large-scale flows. To complete the description of our system and extract all its specificities, we report in figure 5 frequency spectra of the instantaneous velocity for the three components of the flow. Frequency spectra result from time series spanning over 1000 
rotations and are averaged in the spectral domain over 7500 probes, located in the horizontal plane displayed in figure 1.

These frequency spectra can be split into two frequency ranges separated by the cutoff frequency $\omega=2 \Omega$ of the inertial waves. In the range $\omega>2 \Omega$, the dynamics evolve on short time scales and the effect of rotation is moderate. Turbulent energy is nearly isotropically distributed over the three components of the flow (see figure 5). We notice that these high frequency velocities are only weakly affected by the $\beta$-topography: all spectra of both simulations are almost indistinguishable. We observe a $\omega^{-3}$ power law tentatively explained by a Taylor hypothesis: the high-frequency spectrum corresponds to the advection by the large-scale quasi-steady energetic patterns of the direct cascade described in figure 3.

The low frequency range $\omega<2 \Omega$, where rotation dominates (i.e., low Rossby number) and $3 \mathrm{D}$ inertial waves develop, shows the most striking signature of the $\beta$ effect. In simulation DNS2, when no $\beta$-topography is present, rotation acts to separate the horizontal flow from the vertical flow (Campagne et al. 2015). This horizontal anisotropisation is well observed in the frequency spectra figure 5(b) and was already reported in the spatial spectra. In simulation DNS1, where a $\beta$-topography is imposed, the flow undergoes both zonal and horizontal anisotropisation in the range of inertial wave frequencies. Zonal flow dominates over the radial flow component, which in turn, dominates the vertical flow component. These two successive component anisotropies and their frequency spectrum signature are a novelty of our study. Once confirmed from further data analysis, it could establish a new diagnostic of zonostrophic flow tool enabled by our high resolution, turbulent, 3D numerical simulations.

a

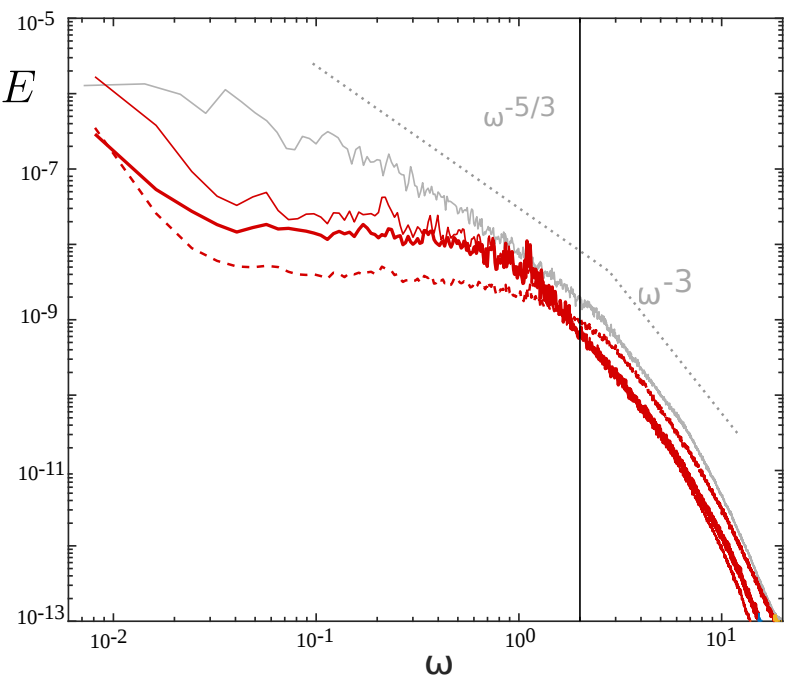

b

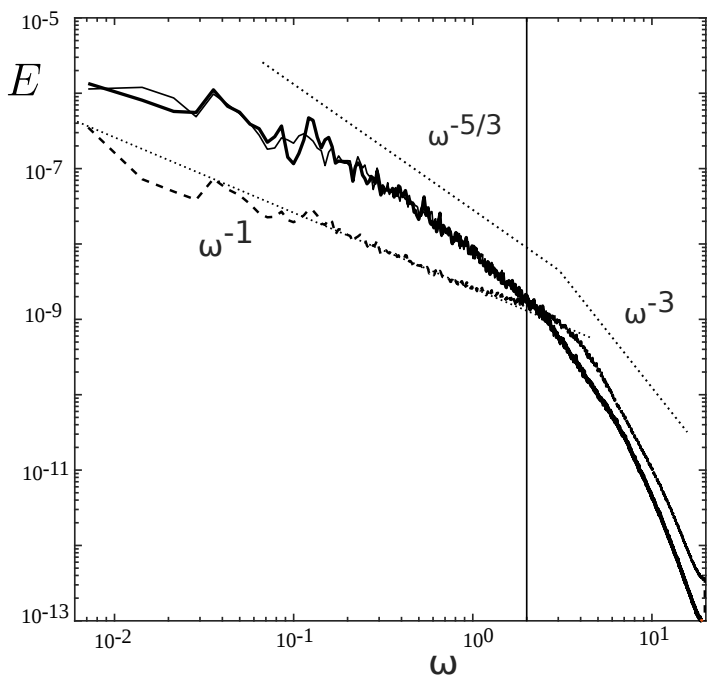

Figure 5. Frequency energy spectra computed from 1000 rotations time series of the zonal (thin solid lines), radial (thick solid lines) and vertical (dashed lines) velocity field components for DNS1 (a) and DNS2 (b). Note that for comparison purpose, we also report on a), the frequency spectra of the zonal component obtained from DNS2 (grey solid line). (Colour online)

A striking feature of these frequency spectra is the energetic plateau within the range of inertial waves frequency, when a $\beta$-topography is imposed (figure $5(\mathrm{a})$ ). The particularity of a $\beta$-topography in rotating system is that it injects potential vorticity and generates Rossby waves that propagate in the zonal direction. Our observations indicate that such a dynamics strongly affects turbulent statistics in a way that energy is equally distributed over 
a large frequency range. Similar features are observed in frequency spectrum of simplified 2D global circulation models used to simulate planetary atmospheres (James et al. 1994). We note that the lowest frequencies in our spectra, which reflect the stationary zonal jets, correspond to a maximum of energy. In the absence of $\beta$-topography, frequency spectra follow a $\omega^{-1}$ power-law for vertical velocity and for the lowest frequencies of horizontal velocities (figure 5(b)). The $\omega^{-1}$ power-law behavior is a prevalent feature in turbulent flows related to the statistical properties of coherent structures (Herault et al. 2015). The horizontal velocities at intermediate frequencies exhibit a nearly $\omega^{-5 / 3}$ power-law, in agreement with the recent laboratory studies of Yarom and Sharon (2014) and Campagne et al. (2015).

\section{Conclusions}

Our phenomenological description of turbulent properties in the so-called zonostrophic regime, and comparatively with the geostrophic (non-zonostrophic) regime, constitutes a coherent and advanced analysis of the first numerical/laboratory model for deep-seated planetary jets presented in Cabanes et al. (2017). We report spectral evidences that an inverse energy cascade occurs under rapid rotation in a 3D-turbulent cylindrical system. We recover several statistics observed in more classical regimes of forced rotating turbulence but also show some new results specific to the case with topography. The presence of a paraboloidal surface shape induces a strong zonal anisotropy of the turbulent flow similarly to deep convective planetary layers. It results that the dominant zonal flow structures into jets and that the statistical flow properties follow the double cascade scenario reminiscent of the 2D turbulence paradigm (Kraichnan 1967), at least for the azimuthal flow velocity. Our high resolution numerical simulations allow us to access the flow in 3D and at all times. We observe that radial and vertical flow components are correlated due to topographical deformation of the top surface. Power spectrum of the vertical velocity field is consequently more energetic in the zonostropic regime where radial motions are converted into vertical motions. This result constitutes an interesting signature of the deep-layer scenario. Ultimately, we report frequency spectra where all three components of the flow are strongly affected by the $\beta$-effect. This feature is probably due to the formation of 3D Rossby waves that are non-existent at constant fluid depth in DNS2. The upcoming data from current and future spacecraft missions will have to be analyzed in view of these spectral analyses that report statistical properties of a deep turbulent planetary layer. Additionally, more theoretical work is requested to explain this $3 \mathrm{D}$ zonostrophic regime that is now accessible to both experimental and numerical investigations.

\section{Acknowledgements}

The authors thank Prof. J. Aurnou (Spinlab, UCLA) for initial suggestions and stimulating discussions, which motivated the present work. This work was supported by the French Agence Nationale pour la Recherche (Program SIMI5 JCJC 2013, Grant No. ANR-13-JS05-0004-01). We also acknowledge support from IDRIS for computational time on Turing (Project No. 100508) and from the HPC resources of Aix-Marseille University (project Equip@Meso No. ANR-10- EQPX-29-01 of the program Investissements d'Avenir). 


\section{References}

Bagenal, F., Dowling, T.E. and McKinnon, W.B., Jupiter: the planet, satellites and magnetosphere, Vol. 1, 2006 (Cambridge University Press).

Berloff, P., Karabasov, S., Farrar, J.T. and Kamenkovich, I., On latency of multiple zonal jets in the oceans. J. Fluid Mech., 2011, 686, 534-567.

Boffetta, G. and Musacchio, S., Evidence for the double cascade scenario in two-dimensional turbulence. Phys. Rev. E, 2010, 82, 016307.

Bouchet, F., Nardini, C. and Tangarife, T., Kinetic theory of jet dynamics in the stochastic barotropic and 2D Navier-Stokes equations. J. Stat. Phys., 2013, 153, 572-625.

Cabanes, S., Aurnou, J., Favier, B. and Le Bars, M., A laboratory model for deep-seated jets on the gas giants. Nature Physics, 2017, 13, 387-390.

Campagne, A., Gallet, B., Moisy, F. and Cortet, P.P., Disentangling inertial waves from eddy turbulence in a forced rotating-turbulence experiment. Phys. Rev. E, 2015, 91, 043016.

Campagne, A., Gallet, B., Moisy, F. and Cortet, P.P., Direct and inverse energy cascades in a forced rotating turbulence experiment. Phys. Fluids, 2014, 26, 125112.

Chertkov, M., Connaughton, C., Kolokolov, I. and Lebedev, V., Dynamics of energy condensation in twodimensional turbulence. Phys. Rev. Lett., 2007, 99, 084501.

Cho, J.Y. and Polvani, L.M., The morphogenesis of bands and zonal winds in the atmospheres on the giant outer planets. Science, 1996, 273, 335.

Christensen, U.R., Zonal flow driven by deep convection in the major planets. Geophys. Res. Lett., 2001, 28, $2553-2556$.

Del Genio, A.D., Achterberg, R.K., Baines, K.H., Flasar, F.M., Read, P.L., Sánchez-Lavega, A. and Showman, A.P., Saturn atmospheric structure and dynamics, 2009 (Springer).

Di Nitto, G., Espa, S. and Cenedese, A., Simulating zonation in geophysical flows by laboratory experiments. Phys. Fluids, 2013, 25, 086602.

Fischer, P.F., Loth, F., Lee, S., Lee, S., Smith, D. and Bassiouny, H., Simulation of high-Reynolds number vascular flows. Computer Methods in Applied Mechanics and Engineering, 2007, 196, 3049-3060.

Fischer, P.F., Lottes, J.W. and Kerkemeier, S.G., nek5000 Web page, Http://nek5000.mcs.anl.gov 2008.

Galperin, B., Sukoriansky, S., Dikovskaya, N., Read, P., Yamazaki, Y. and Wordsworth, R., Anisotropic turbulence and zonal jets in rotating flows with a $\beta$-effect. Nonlinear Process. Geophys., 2006, 13, 83-98.

Galperin, B., Hoemann, J., Espa, S., Di Nitto, G. and Lacorata, G., Anisotropic macroturbulence and diffusion associated with a westward zonal jet: From laboratory to planetary atmospheres and oceans. Phys. Rev. E, 2016, 94, 063102 .

Galperin, B., Sukoriansky, S. and Dikovskaya, N., Geophysical flows with anisotropic turbulence and dispersive waves: flows with a $\beta$-effect. Ocean Dynamics, 2010, 60, 427-441.

Galperin, B., Young, R.M., Sukoriansky, S., Dikovskaya, N., Read, P.L., Lancaster, A.J. and Armstrong, D., Cassini observations reveal a regime of zonostrophic macroturbulence on Jupiter. Icarus, 2014, 229, 295-320.

Gastine, T., Heimpel, M. and Wicht, J., Zonal flow scaling in rapidly-rotating compressible convection. Phys. Earth Planet. Inter., 2014, 232, 36-50.

Heimpel, M., Aurnou, J. and Wicht, J., Simulation of equatorial and high-latitude jets on Jupiter in a deep convection model. Nature, 2005, 438, 193-196.

Heimpel, M., Gastine, T. and Wicht, J., Simulation of deep-seated zonal jets and shallow vortices in gas giant atmospheres. Nature Geoscience, 2016, 9, 19-23.

Heimpel, M. and Gómez Pérez, N., On the relationship between zonal jets and dynamo action in giant planets. Geophys. Res. Lett., 2011, 38.

Herault, J., Pétrélis, F. and Fauve, S., Experimental observation of $1 / \mathrm{f}$ noise in quasi-bidimensional turbulent flows. Europhysics Letters, 2015, 111, 44002.

James, P., Fraedrich, K. and James, I., Wave-zonal-flow interaction and ultra-low-frequency variability in a simplified global circulation model. Quarterly Journal of the Royal Meteorological Society, 1994, 120, 10451067.

Jones, C., A dynamo model of Jupiterś magnetic field. Icarus, 2014, 241, 148-159.

Jones, C.A. and Kuzanyan, K.M., Compressible convection in the deep atmospheres of giant planets. Icarus, 2009, 204, 227-238.

Kaspi, Y., Flierl, G.R. and Showman, A.P., The deep wind structure of the giant planets: Results from an anelastic general circulation model. Icarus, 2009, 202, 525-542.

Kraichnan, R.H., Inertial Ranges in Two Dimensional Turbulence. Phys. Fluids, 1967, 10, 1417-1423.

Marston, J., Chini, G. and Tobias, S., Generalized quasilinear approximation: application to zonal jets. Phys. rev. lett., 2016, 116, 214501.

Maximenko, N.A., Bang, B. and Sasaki, H., Observational evidence of alternating zonal jets in the world ocean. Geophys. Res. Lett., 2005, 32 L12607.

Pouquet, A. and Marino, R., Geophysical Turbulence and the Duality of the Energy Flow Across Scales. Phys. Rev. Lett., 2013, 111, 234501.

Pouquet, A., Sen, A., Rosenberg, D., Mininni, P. and Baerenzung, J., Inverse cascades in turbulence and the case of rotating flows. Physica Scripta, 2013, 2013, 014032.

Read, P., Jacoby, T., Rogberg, P., Wordsworth, R., Yamazaki, Y., Miki-Yamazaki, K., Young, R., Sommeria, 
J., Didelle, H. and Viboud, S., An experimental study of multiple zonal jet formation in rotating, thermally driven convective flows on a topographic beta-plane. Phys. Fluids, 2015, 27, 085111.

Rhines, P.B., Waves and turbulence on a beta-plane. J. Fluid Mech., 1975, 69, 417-443.

Schneider, T. and Liu, J., Formation of jets and equatorial superrotation on Jupiter. J. Atmos. Sci., 2009, 66, $579-601$.

Scott, R.K. and Dritschel, D.G., The structure of zonal jets in geostrophic turbulence. J. Fluid Mech., 2012, 711, $576-598$

Scott, R. and Dritschel, D., Halting scale and energy equilibration in two-dimensional quasigeostrophic turbulence. J. Fluid Mech., 2013, 721, R4.

Sen, A., Mininni, P.D., Rosenberg, D. and Pouquet, A., Anisotropy and nonuniversality in scaling laws of the large-scale energy spectrum in rotating turbulence. Phys. Rev. E, 2012, 86, 036319.

Smith, C.A., Speer, K.G. and Griffiths, R.W., Multiple Zonal Jets in a Differentially Heated Rotating Annulus. J. Phys. Oceanogr., 2014, 44, 2273-2291.

Sukoriansky, S., Dikovskaya, N. and Galperin, B., On the arrest of inverse energy cascade and the Rhines scale. J. Atmos. Sci., 2007, 64, 3312-3327.

Sukoriansky, S., Dikovskaya, N., Grimshaw, R. and Galperin, B., Rossby waves and zonons in zonostrophic turbulence; in AIP Conference Proceedings, Vol. 1439, 2012, pp. 111-122.

Sukoriansky, S., Galperin, B. and Dikovskaya, N., Universal spectrum of two-dimensional turbulence on a rotating sphere and some basic features of atmospheric circulation on giant planets. Phys. Rev. Lett., 2002, 89, 124501.

Vallis, G.K. and Maltrud, M.E., Generation of mean flows and jets on a beta plane and over topography. $J$. Phys. Oceanogr., 1993, 23, 1346-1362.

Yarom, E. and Sharon, E., Experimental observation of steady inertial wave turbulence in deep rotating flows. Nature Physics, 2014, 10, 510-514.

Zhang, Y. and Afanasyev, Y., Beta-plane turbulence: Experiments with altimetry. Phys. Fluids, 2014, 26, 026602 . 